\title{
Texture and Vortex of Rotating Superfluid 3He-A in Parallel-plate Geometry
}

\section{$\operatorname{AUTHOR}(\mathrm{S}):$}

Yamashita, M.; Izumina, K.; Matsubara, A.; Sasaki, Y.; Ishikawa, O.; Takagi, T.; Kubota, M.; Mizusaki, T.

\section{CITATION:}

Yamashita, M. ... [et al]. Texture and Vortex of Rotating Superfluid 3He-A in Parallel-plate Geometry. Journal of Low Temperature Physics 2010, 158(3-4):353-363

ISSUE DATE:

2010-02

URL:

http://hdl.handle.net/2433/128773

\section{RIGHT:}

The original publication is available at www.springerlink.com; この論文 は出版社版でありません。引用の際には出版社版をご確認ご利用くだ さい。; This is not the published version. Please cite only the published version. 


\title{
Texture and Vortex of Rotating Superfluid ${ }^{3} \mathrm{He}-\mathrm{A}$ in Parallel-Plate Geometry
}

\author{
M. Yamashita ${ }^{2}$, K. Izumina ${ }^{1}$, A. Matsubara ${ }^{3}$, Y. Sasaki ${ }^{3}$, O. Ishikawa ${ }^{4}$, T. Takagi ${ }^{5}$, \\ M. Kubota ${ }^{1}$, and T. Mizusaki ${ }^{6}$
}

\begin{abstract}
Unique types of textures and vortices of superfluid ${ }^{3} \mathrm{He}$ in restricted geometries have been discussed. We investigated cw-NMR in rotating ${ }^{3} \mathrm{He}$ A-phase in parallel plate geometries with gaps of $12.5 \mu \mathrm{m}$ at $3.05 \mathrm{MPa}$. We observed a very sharp spectrum at rest which had shifted negatively as $f=f_{L}-0.93 f_{A}(T)$, where $f_{A}(T)$ is the full transverse dipole shift in A-phase. The large negative shift of $0.93 f_{A}(T)$ indicates that $\boldsymbol{l} \perp \boldsymbol{d}$. When we rotated the sample with rotation speed $\Omega$ faster than the critical $\Omega_{F r}$, a new satellite signal appeared nearly at $f_{L}$. The satellite signal intensity increased with increasing $\Omega$, reached a maximum at $\Omega_{c}$ and slowly decreased up to the maximum $\Omega$ of $2 \pi \mathrm{rad} / \mathrm{s}$. When $\Omega$ decreased from the maximum speed, the satellite signal rapidly decreased and disappeared at $5.5 \mathrm{rad} / \mathrm{s}$ down to zero $\mathrm{rad} / \mathrm{s}$. We measured the temperature dependence of the satellite signal intensity, $\Omega_{F r}$ and $\Omega_{c}$. We propose a model for the satellite signal, which is attributed to spin wave in Fréedericksz transition region induced by the counter flow. We also compare the satellite signal with Kee and Maki's bound pair of HQV.
\end{abstract}

Keywords: Superfluid ${ }^{3} \mathrm{He}-\mathrm{A}$, Restricted Geometries, Texture, Half Quantum Vortex, Singular Vortex, Fréedericksz Transition

PACS: $67.30 . \mathrm{H}-$, 67.30.he, $\quad$ 67.30.ht, 67.30.hr

\footnotetext{
${ }^{1}$ Institute for Solid State Physics, The University of Tokyo, Chiba 277-8581, Japan

${ }^{2}$ Department of Physics, Graduate School of Science, Kyoto University, Kyoto 606-8502, Japan,

${ }^{3}$ Research Center for Low Temperatures and Materials Sciences, Kyoto University, Kyoto 606-8502, Japan

${ }^{4}$ Graduate School of Science, Osaka City University, Osaka 558-8585, Japan

${ }^{5}$ Department of Applied Physics, Fukui University, Fukui 910-8507, Japan

${ }^{6}$ Toyota Physical and Chemical Research Institute, Nagakute, Aich 480-1192, Japan

E-mail: mizusaki@scphys.kyoto-u.ac.jp
} 


\section{Introduction}

New types of textures, vortices and coupling effects of textures with flow ${ }^{1}$, which are quite different from those in bulk superfluid ${ }^{3} \mathrm{He}^{2}$, have been discussed for superfluid ${ }^{3} \mathrm{He}$ A phase in parallel plate geometries ${ }^{3-7}$. We have studied $\mathrm{cW}-\mathrm{NMR}$ in rotating superfluid ${ }^{3} \mathrm{He}-\mathrm{A}$ phase confined in a narrow gap between parallel plates, whose separation, $D$, is comparable to or smaller than the dipole coherence length, $\xi_{\mathrm{D}}^{8,9}$. In ${ }^{3} \mathrm{He}-\mathrm{A}$, two vectors describe the order parameter, that is, the orbital part of the order parameter are characterized by $\boldsymbol{l}$, and the spin part of that by $\boldsymbol{d}^{1}$. In the parallel-plate geometry, $\boldsymbol{l}$ points perpendicularly to the surface and $\boldsymbol{d}$ is perpendicular to the applied magnetic field. We apply the field perpendicular to the surfaces of plates, and thus $\boldsymbol{d} \perp \boldsymbol{l}$. This configuration is very different from that of the bulk ${ }^{3} \mathrm{He}-\mathrm{A}$, where $\boldsymbol{l} / / \boldsymbol{d}$. In the parallel plate geometry, the textures are controlled by surfaces, magnetic field and flow (rotation). When the sample is rotated faster than a certain critical counter flow velocity $v_{\mathrm{c}}=\left(\boldsymbol{v}_{\mathrm{n}}-\boldsymbol{v}_{\mathrm{s}}\right)_{\mathrm{c}}$, vortices are introduced and exotic vortices are expected in the parallel plate geometry, where $v_{\mathrm{n}}$ is normal fluid velocity and $v_{\mathrm{s}}$ is superfluid velocity. The flow orients $\boldsymbol{l}$ along $\boldsymbol{v}$, the texture is affected by the flow and may cause Fréedericksz transition ${ }^{1}(\mathrm{FT})$.

There are many types of vortices found in a bulk sample ${ }^{2}$. However, in the parallel-plate geometry with a gap of about $\xi_{\mathrm{D}}, \boldsymbol{l}$ are locked perpendicularly to the surface, a soft core (continuous) vortices observed in a bulk sample cannot exist and a singular (phase) vortex (SV) may be expected. The half quantum vortex (HQV) with a circulation number $N= \pm 1 / 2$ was predicted by Volovik and Mineev ${ }^{3}$ and independently by Cross and Brinkman ${ }^{4}$. The stability of HQV and SV under various conditions of temperature, magnetic field and rotation speed has been discussed ${ }^{5,6,7}$. Recently a bound pair of HQV (b-HQV) and its NMR-signal have been discussed by Kee and $\mathrm{Maki}^{5}$ and were compared with our results ${ }^{8}$. So far, three kinds of vortices, such as SV, HQV and b-HQV, have been predicted in parallel plate geometry.

We investigated cw-NMR spectra of ${ }^{3} \mathrm{He}-\mathrm{A}$ in the parallel-plate geometry with a spacing of $12.5 \mu \mathrm{m}$ in a field perpendicular to the plates ${ }^{8,9}$. We found a new satellite signal, which appeared under rotation for some range of the rotation speed $\Omega$. These results have been compared with Kee-Maki's ${ }^{6}$ phase diagram of b-HQV and free HQV as a function of $\Omega$ and with our model ${ }^{8}$ of the spin wave signal in the region of the flowinduced FT. In this report, we show our spin wave model in some detail and discuss the 
temperature dependences of the critical rotation speed for creation of the new satellite signal, the satellite signal frequency and intensity in comparison with our model.

\section{EXPERIMENTAL RESULTS AND ANALYSIS}

Figure 1 shows the sample cell. The sample cell for parallel plate geometry was made by stacking two kinds of polyimide films with thicknesses of $12.5 \mu \mathrm{m}$ and $25 \mu \mathrm{m}$ shown by (a) and (b). All $12.5 \mu \mathrm{m}$-films (b) have holes of radius $R=1.5 \mathrm{~mm}$ and slits of 0.3 $\mathrm{mm}$-wide channels, which connect other holes of bulk space $(R=1.5 \mathrm{~mm})$. The other 25 $\mu \mathrm{m}$-films (a) have holes $(R=1.5 \mathrm{~mm})$, which serve a bulk part of the sample. The two kinds of film are stacked together one on other to form 110 spaces with gaps $D=12.5 \mu \mathrm{m}$ (see the cross sectional view (d)). Each space (12.5 $\mu \mathrm{m}$ gap and $3 \mathrm{~mm}$ diameter) forms parallel plate geometry and is connected by a $0.1 \mathrm{~mm}$ slit to the bulk sample. These films are packed inside Stycast sample cell (c). We prepared two NMR coils to detect NMR signals for ${ }^{3} \mathrm{He}-\mathrm{A}$ sample in parallel plate geometry and the bulk, shown in (e).

(a)

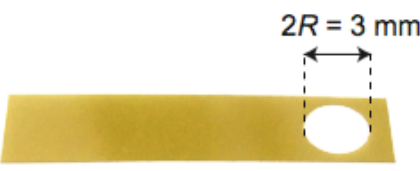

(b)

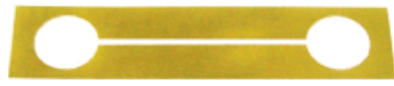

(c)

(d)
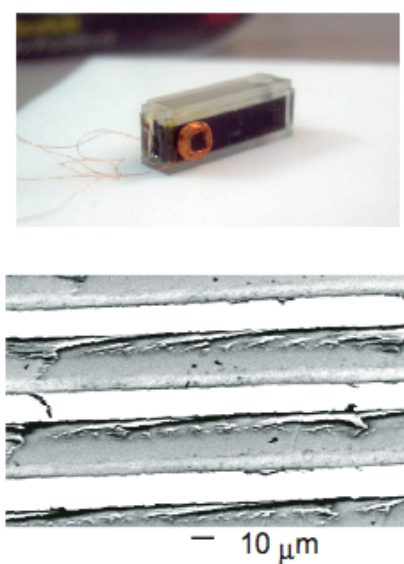

(e)

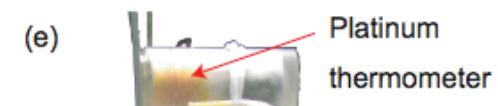

Parallel-plate cell Bulk Space

Fig. 1. (color online) Sample cell. Two kinds of films (a) and (b) to be used for ${ }^{3} \mathrm{He}$ in parallel plate geometry. NMR cell (c) for the parallel-plate sample. (d) Cross sectional view of the stacks of the parallel plates. The sample cell (e) assembled with parallel-plate and bulk samples and PtNMR thermometer. 
The sample was set to the ISSP rotating cryostat and could be rotated up to $2 \pi \mathrm{rad} / \mathrm{s}$. The sample pressure was $3.05 \mathrm{MPa}$ and cw-NMR was taken at the Larmor frequency $f_{L}=$ $869 \mathrm{kHz}$. The NMR magnetic field $H=26.7 \mathrm{mT}$ was applied perpendicularly to the film surface and the rotation axis was also perpendicular to the surfaces.

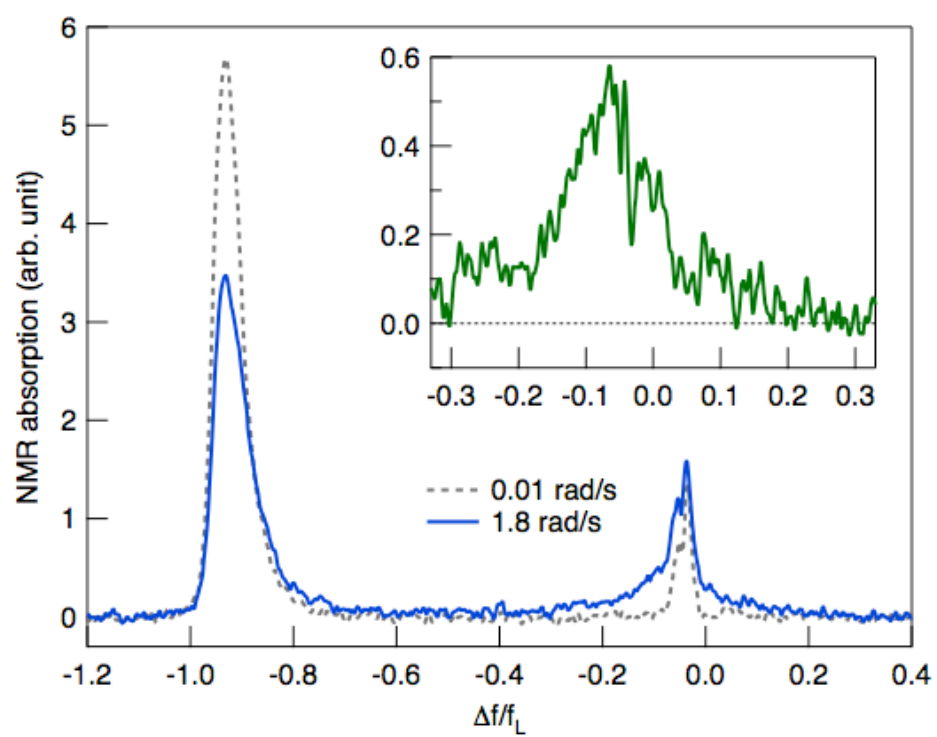

Fig. 2. (color online) Typical NMR absorption spectra as a function of the normalized frequency shift from $f_{L}$. Solid line is spectrum taken at $\Omega=1.80 \mathrm{rad} / \mathrm{s}$ and dotted line is one for $\Omega=0.01$ $\mathrm{rad} / \mathrm{s}$. The satellite signal around $\Delta f / f_{\mathrm{L}}=-0.07$ is shown in inset on an expanded scale.

Figure 2 shows typical cw-NMR absorption spectra for ${ }^{3} \mathrm{He}-\mathrm{A}$ in parallel plate geometry taken at $T=0.81 T_{\mathrm{c}}$, where the transition temperature, $T_{\mathrm{c}}$, was $2.46 \mathrm{mK}$ and the horizontal axis is the frequency shift from the Larmor frequency $f_{L}$ normalized by $f_{\mathrm{A}}(T)=$ $\Omega_{A}(T)^{2} / 2 f_{\mathrm{L}}$ and $\Omega_{A}(T)$ is the longitudinal frequency in A-phase ${ }^{1}$. The sample was cooled down at rest through $T_{\mathrm{c}}$ and the dotted line was taken at a rotation speed $\Omega=0.01 \mathrm{rad} / \mathrm{s}$ and the solid curve at $\Omega=1.80 \mathrm{rad} / \mathrm{s}$. We cooled down the sample with a very small $\Omega$ in order to make sure that the $\boldsymbol{l}$-texture is uniform and regarded it as the sample cooled through $T_{\mathrm{c}}$ at rest. The inset shows the high frequency peak (called a satellite signal) of the spectrum taken at $\Omega=1.8 \mathrm{rad} / \mathrm{s}$ subtracted by the spectrum at rest in the range of $\Delta f / f_{\mathrm{L}}$ between -0.3 and 0.3 . The main peak was fully-negatively-shifted and a higher frequency peak is near $f_{L}$. The small high frequency peak at rest comes from a few layers of solid signals on the whole surface area of films. This is confirmed by the resonance frequency almost at $f_{L}$ and the temperature dependence of the signal intensity, which follows Curie-Weiss law. The negatively-shifted main peak is signal from the uniform texture of ${ }^{3} \mathrm{He}-\mathrm{A}$ in parallel plate geometry. As we started to rotate the sample, the main peak intensity started to decease at $\Omega=1.0 \mathrm{rad} / \mathrm{s}$ while the higher frequency 
increased. The higher frequency peak subtracted by the solid ${ }^{3} \mathrm{He}$ signal at $\Omega=0$ is called a satellite signal. The satellite signal happened to be almost at the same frequency as the solid signal. Total signal intensity of the sum of the main peak and the satellite signal is always conserved under rotation and we can identify the satellite peak induced by rotation.

In uniform texture, the transverse NMR resonance frequency is given by,

$$
f=f_{L}+R_{t}^{2} \times \frac{\Omega_{A}(T)^{2}}{2 f_{L}}=f_{L}+R_{t}^{2} \times f_{A}(T),
$$

where $R_{t}^{2}=\cos (2 \theta)$ and $\theta$ is the angle between $\boldsymbol{l}$ and $\boldsymbol{d}^{1}$. In bulk sample, $\theta=0$ and $f_{A}(T)$ is measured from bulk A-phase NMR. The angle $\theta$ in parallel plate geometry is expected to be $\pi / 2$ and $R_{t}^{2}$ is -1 . The temperature dependence of the main peak is given by $f=f_{L}-0.93 f_{A}(T)$ by using measured values of $f_{A}(\mathrm{~T})$ in the bulk sample. The sharpness of the main peak and $R_{t}^{2}=-0.93$ indicates that $l$ is oriented almost perpendicular to $\boldsymbol{d}$ and films are stacked uniformly and parallel to each other (see also the cross sectional view (d) in Fig. 1).

The satellite signal introduced by rotation appears at $R_{t}^{2}=-0.07$. Since the solid signal appears almost at the same frequency as that for the satellite signal, we define the intensity of the satellite signal $I_{\mathrm{s}}(\Omega)$ as,

$$
I_{s}(\Omega)=\int_{f_{L}-f_{A} / 3}^{f_{L}+f_{A} / 3}(I(f . \Omega)-I(f, 0)) d f
$$

where $I(f, \Omega)$ is the NMR intensity at a frequency $f$ under $\Omega$, as shown in Fig. 1 and the solid signal is subtracted to get the satellite signal. In Fig. 3, the normalized $I_{s}(\Omega)$ by $I_{\text {total }}$, denoted as $I_{s}^{\prime}(\Omega)$, is plotted as a function of $\Omega$, where $I_{\text {total }}$ is a constant for any $\Omega$. The interval of the integrals in Eq. (2) is somewhat arbitrary and is chosen to cover the line width of the satellite signal near $f_{L}$, as shown in the inset of Fig. 2. 


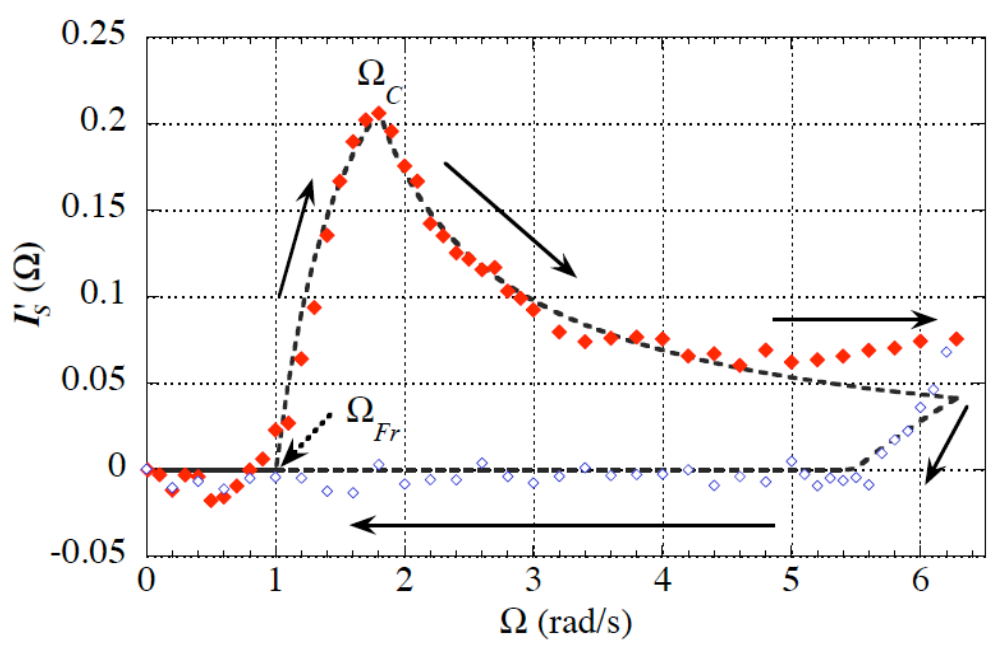

Fig. 3. (color online) Normalized satellite signal intensity as a function of $\Omega$. Dotted line is the spin wave intensity predicted by the FT model.

This data was taken at $T=0.81 T_{\mathrm{c}}$ when the sample was cooled through $T_{\mathrm{c}}$ at rest and the sample started to rotate at $T<T_{\mathrm{c}}$, as shown by arrows in the direction for data to be taken. In acceleration from $\Omega=0$, no change was observed in the NMR spectrum and no satellite appears below $\Omega_{F r}=1 \mathrm{rad} / \mathrm{s}$. Above $\Omega_{F r}$, the satellite signal was observed nearly at $f_{L}$, and increased rapidly up to $\Omega_{c}=1.8 \mathrm{rad} / \mathrm{s}$. The satellite signal had a maximum at $\Omega_{c}$, decreased gradually as $\Omega$ increased and became almost constant above $4 \mathrm{rad} / \mathrm{s}$ up to $2 \pi \mathrm{rad} / \mathrm{s}$. When $\Omega$ was deceased from $2 \pi \mathrm{rad} / \mathrm{s}$, the satellite signal deceased rapidly and disappeared at $5.5 \mathrm{rad} / \mathrm{s}$ down to $\Omega=0$.

We define here $\Omega_{F r}$ at which the satellite signal appears and $\Omega_{\mathrm{c}}$ at which the satellite signal takes a maximum value. The $I_{\mathrm{s}}(\Omega)$ vs. $\Omega$ shows a large hysteresis. It should be noted that when $\Omega$ changes between 0 and $\Omega_{\mathrm{c}}, I_{\mathrm{s}}(\Omega)$ is reversible. But once $\Omega$ increases beyond $\Omega_{\mathrm{c}}, I_{\mathrm{s}}(\Omega)$ is not reversible any more and shows the hysteresis. $\quad$ It is also noted that when the sample is cooled through $T_{\mathrm{c}}$ with rotation speed of $\Omega=2 \pi \mathrm{rad} / \mathrm{s}$, the satellite signal was not observed and when $\Omega$ was decreased down from $2 \pi \mathrm{rad} / \mathrm{s}$ to zero, no change in spectrum and no satellite signal were observed down to $\Omega=0$.

It may be important to investigate temperature dependence of the satellite signal to find the origin of the satellite signal. The satellite-signal frequency depends on $T$ but $R_{t}^{2}=-0.07$ do not depend on $T$. We show $\Omega_{F r}$ and $\Omega_{c}$ as a function of $T$ in Fig. 4. 


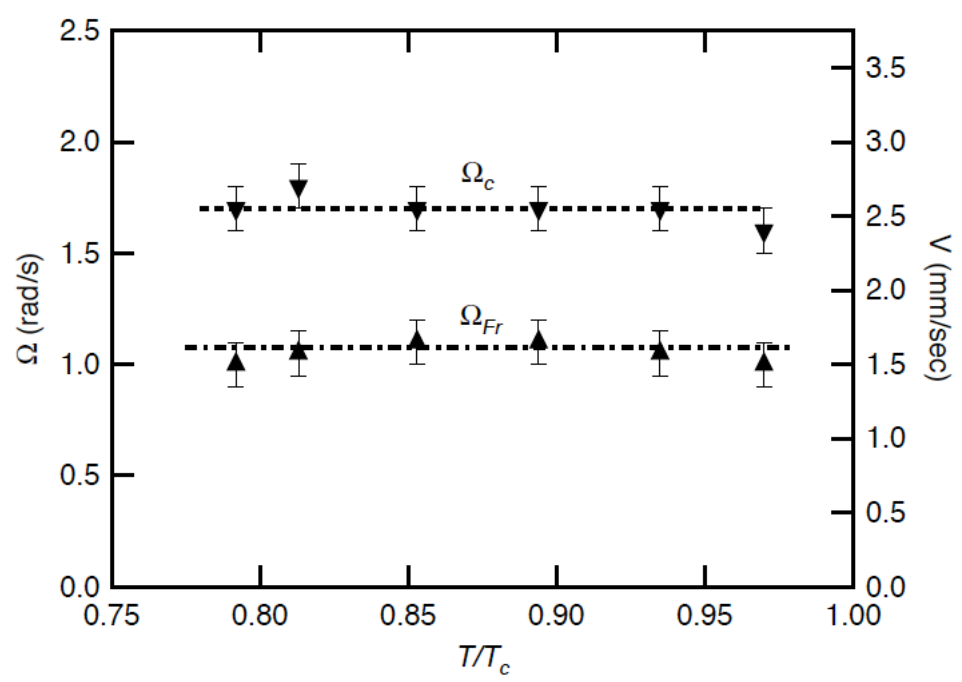

Fig. 4. Temperature dependence of $\Omega_{F r}$ and $\Omega_{c}$.

The vertical axis on left-hand-side is the velocity at the edge of the sample and is obtained by $V=R \Omega=1.5 \mathrm{~mm} \Omega$. Both $\Omega_{c}$ and $\Omega_{F r}$ do not depend on $T$ and the average values are $\Omega_{c}=1.70 \mathrm{rad} / \mathrm{s}\left(V_{\mathrm{c}}=2.6 \mathrm{~mm} / \mathrm{s}\right)$ and $\Omega_{F r}=1.1 \mathrm{rad} / \mathrm{s}\left(V_{\mathrm{Fr}}=1.7 \mathrm{~mm} / \mathrm{s}\right)$.

Figure 5 shows the temperature dependence of $I_{\mathrm{s}}(\Omega)$. Since $\Omega_{\mathrm{c}}$ and $I_{\text {total }}$ do not depend on $T$, we pick up values of $I_{\mathrm{s}}(\Omega)$ at $\Omega=\Omega_{c}$ and $I_{\mathrm{s}}\left(\Omega_{\mathrm{c}}\right)=I_{\mathrm{s}}\left(\Omega_{c}\right) / I_{\text {total }}$ are plotted against $T / T_{\mathrm{c}}$. The value of $I_{\mathrm{s}}^{\prime}\left(\Omega_{\mathrm{c}}\right)$ are 0.22 at $T / T_{\mathrm{c}}=0.8$ and tends to become zero at $T_{\mathrm{c}}$. It should be noted that $I_{\mathrm{s}}^{\prime}\left(\Omega_{\mathrm{c}}\right)$ and thus $I_{\mathrm{s}}(\Omega)$ depend on $T$ even though all quintiles such as $\Omega_{F r}, \Omega_{c}$ and $I_{\text {total }}$ are independent of $T$.

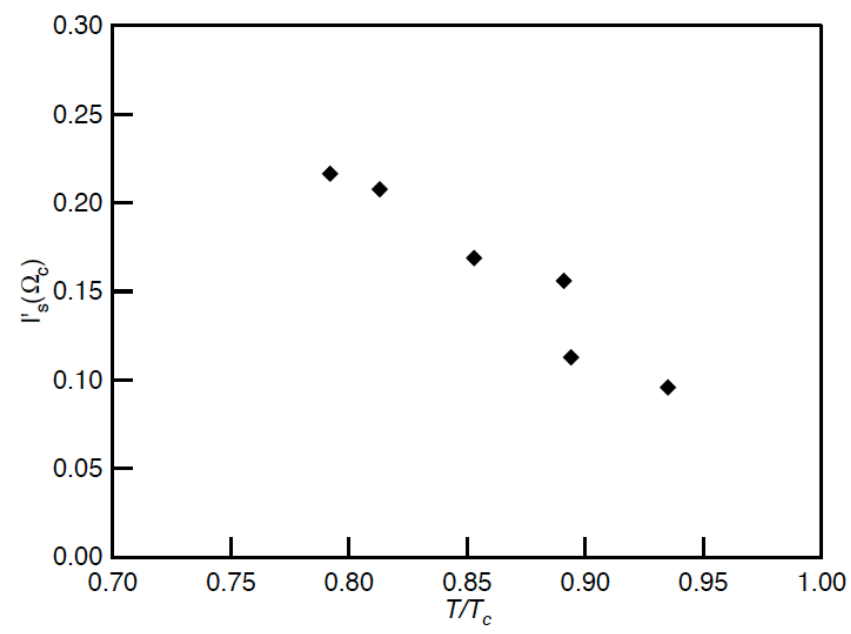

Fig. 5. Temperature dependence of the normalized satellite intensity at $\Omega_{\mathrm{c}}$.

\section{DISCUSSION}


When we rotated ${ }^{3} \mathrm{He}-\mathrm{A}$ in the parallel plate geometry, rotation would cause the coupling of the texture with flow and introduction of quantized vortices. Regarding vortices, three kinds of vortices, SV, HQV and b-HQV, have been proposed. No NMR signal is expected to be observed for SV and HQV. Kee and Maki suggested that HVQ form bound pairs at low vortex density, which would cause a large spin wave signal trapped by b-HQV. In this section, we introduce our model to explain the observed satellite signal, which is attributed to the spin wave signal trapped in the dipole potential of the flowinduced FT region. We also compare our result with Kee and Maki's b-HQV model.

\subsection{Flow-induced FT and spin wave in the FT region}

When the sample is rotated, the normal component of the superfluid rotates as a solid body and the normal fluid velocity is written as $v_{n}=r \Omega$, where $r$ is distance from the rotation axis. Because of the anisotropic energy gap of A-phase along $l$, normal flow tends to orient $\boldsymbol{l}$ parallel to the flow. Between parallel plates, $\boldsymbol{l}$ texture under a normal flow is determined by a balance between orientational forces (the boundary condition of $\boldsymbol{l}$ on the surface, the flow and the dipole interaction and the bending energy $)^{1}$. Under such competitions, it is well known that there is a threshold in the counterflow velocity $v_{F r}$, above which the system undergoes a Fréedericksz transition (FT) from a uniform texture to a non-uniform one. We numerically calculate FT and spin wave trapped by dipole potential caused by FT transition. Our model is as follows: The parallel plate has a gap of $D$ which is comparable to $\xi_{D}$ and the plate is in $x-y$ plane and the $z$-axis is perpendicular to the plate. The normal fluid (or counter flow) flows with velocity $v$ along x-axis. We fix $\boldsymbol{d}$ along $\mathrm{x}$-direction to minimize the magnetic field energy. The $\boldsymbol{l}$ vector is given by $l=\sin \beta e_{x}+\cos \beta e_{z}$, where $\beta$ is a angle between the $\mathrm{z}$-axis and $l$. We determine the $l$-texture by minimizing the following free energy ${ }^{10}$ as,

$F=\int_{-D / 2}^{D / 2} d z\left[\frac{1}{2}\left\{\left(\rho_{\perp} v^{2}+\left(\rho_{/ /}-\rho_{\perp}\right) v^{2} \sin ^{2} \beta+\left(K_{s}+K_{b}\right)\left(\frac{\partial \beta}{\partial z}\right)^{2} \cos ^{2} \beta\right\}-\frac{g_{D}}{2} v^{2} \sin ^{2} \beta\right]\right.$,

where the 1 st term is the sum of kinetic energy of flow and bending energy and the 2nd term is the dipole energy. We minimize the above free energy subjecting the boundary condition that $\boldsymbol{l}$ is perpendicular to the wall and determine the $\boldsymbol{l}$-texture as a fixed normal flow velocity $\boldsymbol{v}$. The distribution of $\boldsymbol{l}$ is determined as a function of $\mathrm{z}$ and the dipole energy $\left(\propto(d \cdot l)^{2}=\sin ^{2} \beta(\mathrm{z})\right)$ has position dependence. The $l$ around $\mathrm{z}=0$ (at the 
middle of the gap) can be deflected along the flow when the normal flow exceeds a critical velocity, $v_{F r}$. Since $D$ is comparable to $\xi_{\mathrm{D}}$, non-uniform dipole field can trap a spin wave and a new satellite signal of the spin wave appears in NMR spectrum.

In Fig. 6, the inset shows the spin wave frequencies as a function of $v$ for various values of a parameter, $D / \xi_{D}$. The FT appears at $v=v_{F r}$ and its spin wave frequency rapidly increases from $R_{t}^{2}=-1$ and then is saturated at some value. The results are very sensitive to the choice of $\xi_{D}$. The calculated critical velocity $v_{\mathrm{Fr}}$ is about $3 \mathrm{~mm} / \mathrm{s}$ and the saturated value of the frequency is about $R_{t}^{2} \sim 0\left(f \sim f_{L}\right)$ for $\xi_{D}=10 \mu \mathrm{m}$. Figure 6 shows the calculated NMR spectrum by using the inset of Fig. 5 for a case of $\Omega=1.8 v_{\mathrm{Fr}} / R$, where $v(r)=r \Omega$ for $0<r<R$ and $R=1.5 \mathrm{~mm}, D=12.5 \mu \mathrm{m}$ and $\xi_{D}=10 \mu \mathrm{m}$. We identified the onset value of $\mathrm{v}$ for the satellite signal as $v_{F r}$ and $v_{F r}=R \Omega_{F r}=1.5 \mathrm{~mm} / \mathrm{s}$ and $\Omega_{F r}=1.0 \mathrm{rad} / \mathrm{s}$ was found (see Fig. 3). The spin wave frequency was $R_{t}^{2}=-0.07$ (see

Fig.2). The agreement with the experiment is good for the choice of $\xi_{D}=10 \mu \mathrm{m}$.

Now we consider the spin wave signal intensity and the hysteresis as a function of $\Omega$, as shown in Fig. 2

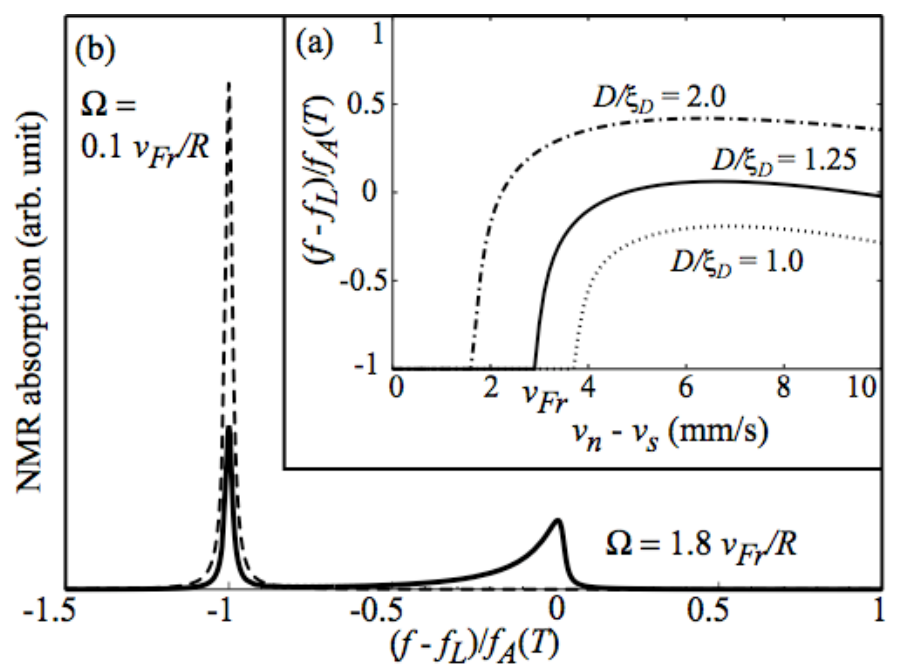

Fig. 6. The FT-induced spin wave frequency predicted for $\xi_{D}=10 \mu \mathrm{m}$. The inset is the spin wave frequency in the FT region as a function of the counter flow velocity for various values of $D / \xi_{\mathrm{D}}$. 
(a)

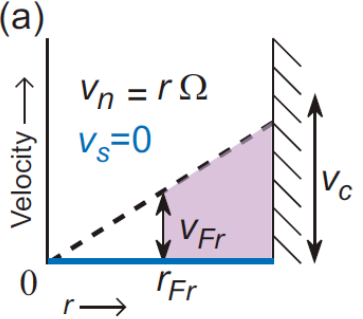

(c)

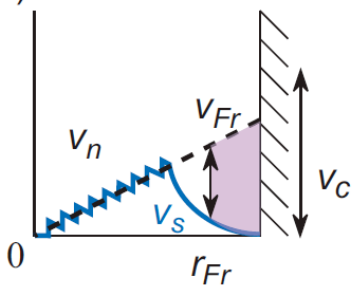

(b)

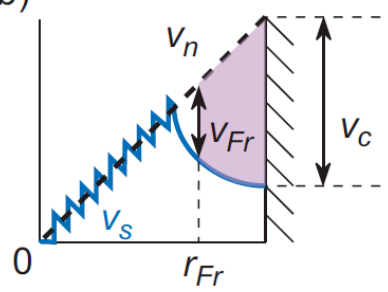

(d)

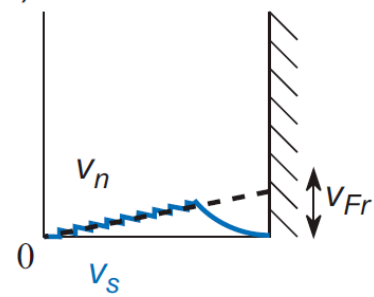

Fig. 7. (color online) Flow profile of the normal fluid velocity $v_{\mathrm{n}}(r)$ (dotted line) and the superfluid velocity $v_{\mathrm{s}}(r)$ (solid curve) as $\Omega$ increases in (a) and (b) and then decreases in (c) and (d) after it reaches a maximum $\Omega$.

Figure 7 schematically shows flow patterns of normal fluid velocity (dotted line) $v_{n}(\mathrm{r})=r \Omega$ and superfluid velocity (solid curve) $v_{s}(\mathrm{r})$ in the rotational direction in the parallel plate geometry. The region where $v=v_{n}-v_{s}>v_{F r}$ is illustrated as a shaded area. In acceleration: (a) For $\Omega>\Omega_{F r}$, FT region proceeds inward as rotation speed increases, where $v_{\mathrm{s}}=0$. (b) For $\Omega>\Omega_{c}$, vortices are created at $r=$ $R$ and form a vortex cluster around the rotation center, which expands outward as $\Omega$ increases to keep $v_{n}-v_{s}=v_{c}$ at $r=R$. In deceleration: (c) vortex cluster expands with keeping the number of vortices. The FT region quickly shrinks as rotation speed decreases. (d) there is no FT region. A similar model of vortex distribution in bulk liquid ${ }^{11}$ is used to evaluate the superfluid flow effect on $I_{\mathrm{s}}(\Omega)$.

Above $\Omega_{F r}$ in acceleration, FT starts from $r=R$ and expanded inward in the acceleration as $1-\left(r_{F r} / R\right)^{2}$ as illustrated in Fig. 7 (a), where $r_{F r}=R \Omega_{F r} / \Omega$ is the inner edge of the FT region. The critical rotation speed at the peak of $\left.I_{\mathrm{s}}(\Omega)\right), \Omega_{\mathrm{c}}$ $=1.85 \mathrm{rad} / \mathrm{s}$, can be attributed to a critical velocity for creation of vortices in the parallel-plate geometry at the edge of the sample. Here we do not consider any pinning effect on vortices between plates. Vortices produce superfluid flow, $v_{\mathrm{s}}$, and reduce the counterflow velocity. In this model, vortices come into the superfluid so that the counterflow at the wall is maintained as $v_{\mathrm{n}}(R)-v_{\mathrm{s}}(R)=v_{\mathrm{c}}$, where $v_{\mathrm{c}}=R \Omega_{\mathrm{c}}$ is the critical velocity for vortex, and these vortices form a cluster around the rotation center. Inside the cluster, there is no macroscopic 
counterflow and thus no FT region. While the outside of the cluster region, the counterflow is given as $v_{\mathrm{n}}(\mathrm{r})-v_{\mathrm{s}}(\mathrm{r})=r \Omega-\kappa N_{\mathrm{v}} / 2 \pi \mathrm{r}$, where $\kappa$ is the quantum of circulation and $N_{\mathrm{v}}$ is the number of vortices, the FT region is reduced in area by the vortices. In deceleration, the vortex cluster expands with keeping the vortex number [Fig. 7 (c)], and the FT region disappears in further deceleration [Fig. 7 (d)].

In Fig. 3, we plot the area where the counterflow exceeds $v_{F r}$ as a function of $\Omega$ as a dashed line with a fitting parameter $\alpha$ as $\left.\alpha\left[1-\left(r_{F r}\right) / R\right]^{2}\right]$. This line describes $I_{\mathrm{s}}^{\prime}(\Omega)$ very well by adjusting $\alpha=0.3$. This good coincidence strongly supports our interpretation that FT is taking place above $\Omega_{F r}$ by counter flow and the counter flow effect is reduced by vortices which appear above $\Omega_{\mathrm{c}}$.

NMR spectrum taken in deceleration between $\Omega=5.5$ and $0 \mathrm{rad} / \mathrm{s}$ correspond to the case shown in Fig. 7 (d), where the counter flow velocity should be smaller than $v_{\mathrm{Fr}}$ and therefore there is no FT region, and should be identical to the initial spectrum shown by a dotted line in Fig. 1 taken at $\Omega=0 \mathrm{rad} / \mathrm{s}$. However, there should be lots of vortices trapped in this case but no vortex signal was observed. This result indicates that vortices in the parallel plate geometry were not observed by NMR and vortices in parallel-plate geometry are quite different from soft-core vortices observed in the bulk A-phase ${ }^{2}$. This implies that the vortices may be SV or HQV (not b-HQV predicted by Kee and Maki ${ }^{5}$ ). It is noted that the main peak signal in the case of Fig. 7(d) was identical to that without vortices and did not show any broadening of the line width under influence of vortices. This also implies that vortices in the parallel plate geometry do not have soft cores.

One of the results which does not agree with FT-induced spin wave model is that we need a large reduction factor $\alpha=0.3$ to explain the size of $I_{s}^{\prime}(\Omega)$. The spin-wave wave function in the FT region is uniform across the gap of the parallel plate and we expect the fitting parameter $\alpha$ should be about 1, which disagree with the observed value of 0.3 . Furthermore, the only fitting parameter in the calculation is $\xi_{\mathrm{D}}$, which is temperature-independent. Therefore FT-induced spin wave model does not predict any strong temperature dependence of $I_{\mathrm{s}}(\Omega)$, which contradicts the result shown in Fig. 4. Further studies would be needed to reveal the nature of vortices and the satellite signal which we believe to be attributed to the FT-induced spin wave signal. 


\section{2. Kee and Maki's b-HQV model and its satellite signal}

Kee and Maki studied stability conditions among singular vortex, HQV and b-HQV as a function of rotation speed where average distance between two vortices $2 \mathrm{a}$ is given by $1 /\left(\pi a^{2}\right)=2 \Omega / N \kappa$, where $\kappa$ is quantum circulation and $N$ the number of quanta per vortex. They predicted that, (a) The SV is unstable against HQV if $\Omega<5 \times 10^{6} \mathrm{rad} / \mathrm{s}$. (b) The b-HQV is most stable for small $\Omega$ with a low density of vortices. (c) For $\Omega>$ $\Omega_{\mathrm{c}}$, free HQV is more stable than b-HQV. They estimate $\Omega_{\mathrm{c}}=2 \sim 12 \mathrm{rad} / \mathrm{s}$. (d) the bHQV are detectable by NMR.

They interpreted the result of satellite signal $I_{\mathrm{s}}^{\prime}(\Omega)$ shown in Fig. 3 as follows. The $\Omega_{\mathrm{Fr}}$ corresponds to some kind of the critical speed of rotation for vortex nucleation and for $\Omega>\Omega_{\mathrm{Fr}}$, b-HQV are introduced and the vortex density increases as $\Omega$ increases. At $\Omega=\Omega_{\mathrm{c}}$, b-HQV breaks into free HQV. Since free HQV does not contribute to NMR signal, $I_{\mathrm{s}}{ }(\Omega)$ decreases as the number of b-HQV decreases. They pointed out that the spin wave is trapped at b-HQV and the observed intensity $I_{\mathrm{s}}^{\prime}(\Omega)$ and frequency of the b-HQV spin wave agree with our observation.

Their result of the critical values of pair-braking speed of rotation, spin wave frequency and intensity does not strongly depend on $T$ so that they could not explain the temperature dependence of $I_{\mathrm{s}}^{\prime}(\Omega)$ shown in Fig. 5. Their model of b-HQV does not agree with the hysteresis curve in Fig. 3, particularly in the decelerated process where $I_{\mathrm{s}}^{\prime}(\Omega)$ quickly disappeared down to $\Omega=0$. It is difficult to explain the historydependent result for $I_{\mathrm{s}}^{\prime}(\Omega)$ by this model unless some pinning effects of vortices are introduced.

\section{Summary}

We have measured NMR on ${ }^{3} \mathrm{He}-\mathrm{A}$ under rotation in parallel plate geometry whose gap was comparable to $\xi_{D} \sim 10 \mu \mathrm{m}$. We found a very narrow main peak at rest with $R_{t}^{2}=$ -0.93 (almost full negative shift), that indicates the sample had a uniform texture with the configuration of $\boldsymbol{l} \perp \boldsymbol{d}$. When we rotated the sample, a new satellite peak appeared at $R_{t}^{2}=-0.07$ for $\Omega>\Omega_{F r}=1.1 \mathrm{rad} / \mathrm{s}$. The satellite intensity $I_{\mathrm{s}}^{\prime}(\Omega)$ reached a maximum at $\Omega_{C}=1.7 \mathrm{rad} / \mathrm{s}$ and decreased gradually as $\Omega$ was increased. When $\Omega$ decreased from the maximum rotation speed of $2 \pi \mathrm{rad} / \mathrm{s}, I_{\mathrm{s}}(\Omega)$ decreased rapidly and 
disappeared below $5.5 \mathrm{rad} / \mathrm{s}$ and down to $0 \mathrm{rad} / \mathrm{s}$. The quantities, $\Omega_{F r}, \Omega_{c}$ and $R_{t}^{2}$, did not depend on $T$ but $I^{\prime}{ }_{\mathrm{s}}(\Omega)$ depended on $T$.

Based on hysteresis of the satellite signal against $\Omega$, we propose the spin wave model in the flow-induced FT region. We calculated the flow-induced FT and the spin wave frequency and intensity trapped in the FT region. The spin wave frequency or $R_{t}^{2}$ and the onset velocity of the FT, $V_{\mathrm{c}}$, agree well with experimental result when we chose $\xi_{D}=10 \mu \mathrm{m}$. In order to explain the hysteresis in $I_{\mathrm{s}}(\Omega)$, we introduced the vortex nucleation critical rotation speed $\Omega_{\mathrm{c}}$ and velocity $v_{\mathrm{c}}=R \Omega_{\mathrm{c}}$. For $\Omega>\Omega_{\mathrm{c}}$, the introduction of vortices reduces the counter flow velocity and thus decreases $I_{\mathrm{s}}(\Omega)$. This model agrees well with experimental results except for the temperature dependence $I_{\mathrm{s}}(\Omega, T)$ and the size $\alpha=0.3$.

We compared our result with Kee and Maki's model. They claimed that the satellite signal comes from b-HQV. They identified that $\Omega_{\mathrm{c}}$ is the pair-breaking critical rotation and b-HQV becomes non-bound free $\mathrm{HQV}$ for $\Omega>\Omega_{\mathrm{c}}$. They estimated $\Omega_{\mathrm{c}}$ to be $2 \sim 12 \mathrm{rad} / \mathrm{s}$ and a size of signal $I_{\mathrm{s}}(\Omega)$, which agrees with our results. However their model does not explain the hysteresis and temperature dependence of $I_{\mathrm{s}}^{\prime}(\Omega)$.

Acknowledgement We acknowledge that this experiment was carried out by using facilities of the Institute for Solid State Physics, The University of Tokyo and was partially supported by a Grant-in-Aid for Scientific Research on Priority Areas by Ministry of Education, Culture, Science, Sport and Technology, Japan.

\section{REFERENCES}

1. D. Vollhardt and P. Wölfle, The Superfluid Phase of Helium three (Taylor \& Francis, London, 1990).

2. O. V. Lounasmaa and E. Thuneberg, Proc. Natl. Acad. Sci. U.S.A. 96, 7760 (1999).

3. G. E. Volovik and V. P. Mineev, JETP Lett., 121, 561 (1976).

4. M. C. Cross and W. F. Brinkman, J. Low Temp. Phys., 27, 683 (1977).

5. H.-Y. Kee and K. Maki, Europhys. Lett., 80, 46003 (2007).

6. T. Kawakami, T. Tsutsumi, and K. Machida, Phys. Rev. B 79, 092506 (2009).

7. V. Vakaryuk and A. Leggett, arXiv:0905.2631v1.

8. M. Yamashita et al., Phys. Rev. Lett,. 101. 025302 (2008).

9. M. Yamashita et al., AIP Conference Proceedings, 850, 185 (2006).

10. M. C. Cross, J. Low Temp. Phys. 21. 525 (1975).

11. P. M. Walmsley, D. J. Cousins, and A. I. Golov, Phys. Rev. Lett. 91, 225301 (2003). 